\title{
JURI SAYS: An Automatic Judgement Prediction System for the European Court of Human Rights
}

\author{
Masha MEDVEDEVA ${ }^{\mathrm{a}, \mathrm{b}, 1}$, Xiao XU $^{\mathrm{a}}$, Martijn WIELING $^{\mathrm{a}}$ and Michel VOLS ${ }^{\mathrm{b}}$ \\ ${ }^{a}$ Center for Language and Cognition, University of Groningen, The Netherlands \\ ${ }^{\mathrm{b}}$ Department of Legal Methods, University of Groningen, The Netherlands
}

\begin{abstract}
.
In this paper we present the web platform JURI SAYS that automatically predicts decisions of the European Court of Human Rights based on communicated cases, which are published by the court early in the proceedings and are often available many years before the final decision is made. Our system therefore predicts future judgements of the court. The platform is available at jurisays.com and shows the predictions compared to the actual decisions of the court. It is automatically updated every month by including the prediction for the new cases. Additionally, the system highlights the sentences and paragraphs that are most important for the prediction (i.e. violation vs. no violation of human rights).
\end{abstract}

Keywords. European Court of Human Rights, machine learning, web platform

\section{Introduction}

In recent years, the use of machine learning for predicting judicial decisions has become more popular $[1,2,3,4,5,6,7]$ as these methods are able to detect patterns in increasingly large legal datasets. In this paper we introduce an online platform, JURI SAYS, which automatically retrieves legal documents from the European Court of Human Rights (ECtHR) database, and subsequently predicts the judgements of the cases on the basis of information which was available before the judgement was made. In addition to predicting decisions, JURI SAYS identifies and highlights sentences that were most important for its prediction.

The JURI SAYS system can roughly be divided into three parts: 1) a database, 2) a machine learning system, and 3) a web platform. Each part is independent from the others and offers a set of Application Programming Interfaces (APIs) to add flexibility for the future, allowing (for example) more documents to be added, new machine learning models to be included, or adjusting the interface. Before discussing the architecture of the system, however, some background on the legal data underlying our system is necessary.

\footnotetext{
${ }^{1}$ Corresponding Author: Masha Medvedeva, Center for Language and Cognition Groningen, Oude Kijk in 't Jatstraat 26, 9712 EK Groningen, the Netherlands; Email: m.medvedeva@rug.nl
} 


\section{Data}

The European Court of Human Rights is an international court established in 1959 that deals with individual and State applications claiming a violation of various rights laid out in the European Convention on Human Rights (ECHR) [8, 9, 10]. Applications are always brought by an individual against a State or multiple States that have ratified the ECHR, with rare exception of State against State cases.

To our knowledge, all previous research on predicting decisions of the ECtHR used various parts of the final decision published by the court. In this work we refrain from using these documents for prediction, because when they are compiled the final decision is already known and the text (even parts which do not contain the judgement itself) may reflect that decision [7]. Fortunately, however, the court publishes multiple documents at various stages of the proceedings.

Once the application is made by the alleged victim, and fits the formal admissibility criteria, the court often communicates the facts of the case to the State against which there is a complaint. It also poses some questions to the State, so that the State may corroborate or deny the allegations. These documents are labeled as Communicated cases on the HUDOC website. ${ }^{2}$ JURI SAYS predicts the decisions on the bases of these documents. Once the case is communicated, it goes through an admissibility stage, where it is evaluated based on merit. The clear-cut cases with no violation are then found inadmissible, the rest move to the next stage, where the judgement is made, and the final document with facts, arguments and the decision is produced.

\section{JURI SAYS}

\subsection{Database}

Our database only includes documents in English. Every month new documents are downloaded and a new machine learning model to predict the ECtHR decisions of that month is trained (see below). At present our database contains 4929 communicated cases with their associated decision. While the predictions are only based on the communicated cases, we also include information from cases from the last ten years that were not communicated to increase the amount of data available to train our model. For those cases, we only extract the "Facts" part from the final document with the judgement [7].

Our system automatically extracts the raw text of the communicated cases from the database of the ECtHR, in addition to some metadata, such as the decisions (for admissibility cases and judgements), data, parties, articles involved, et cetera. The decisions are then associated to the communicated cases on the basis of the application number.

\subsection{Machine learning system}

Every month, after downloading the new documents, the system behind our web platform JURI SAYS carries out three tasks. It first trains a new machine learning model (introduced in Medvedeva et al. [7]) on the basis of all data excluding the data from the most recent month. Then it predicts the judicial decision for the cases of the most recent month on

\footnotetext{
${ }^{2}$ https://hudoc.echr.coe.int
} 


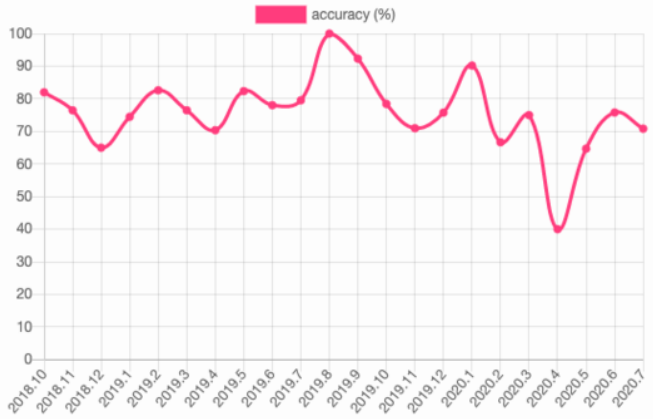

Figure 1. Accuracy of JURI SAYS over the past two years predicting future judgements.

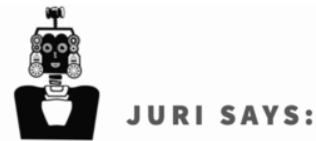

\section{I correctly predicted that there's no violation of human rights in ALBERT AND OTHERS v. HUNGARY.}

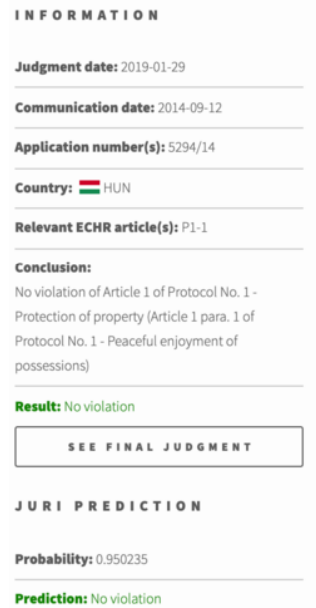

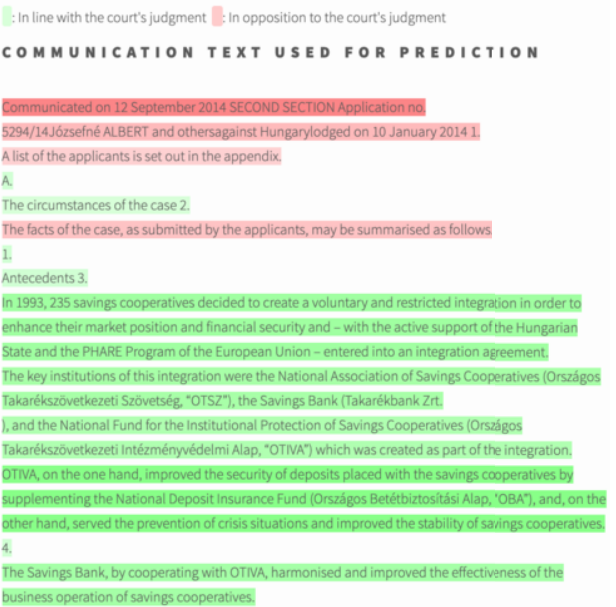

Figure 2. An example of a correctly predicted case by JURI SAYS with highlighted sentences.

the basis of the newly-created model. The performance (accuracy) of JURI SAYS for each month during the last two years can be found in Figure 1. Finally, for each sentence in the text of the communicated case, it identifies how strongly it is related to the actual judgement of the court (by estimating the probability of the sentence belonging to a case with a violation versus to a case without a violation of human rights; see also Figure 2).

\subsection{Web platform}

JURI SAYS is the web platform of our system presenting the results of applying our machine learning system to the extracted data of the ECtHR. JURI SAYS is updated every 
month by publishing the predictions for the most recent ECtHR cases. It also offers a list of all historical cases that may be ordered or filtered by date or article involved. For every single case there is a separate page that offers more detailed information, including the predicted outcome of the case, together with an associated probability of that predicted outcome, and the actual judgement of the court. For each sentence in the text of the communicated case, the predicted label and associated probability is shown when the mouse pointer is hovered over a sentence. Sentences which are highlighted in green are consistent with the court's actual decision, those in red are more likely to be associated with the opposite decision. See Figure 2 for an example. The intensity of the colour reflects how strongly associated the sentences are with the respective decisions.

\section{Conclusion}

In this paper, we have presented JURI SAYS, an automatic judgement prediction system for the ECtHR. Our system uses automatically extracted textual information from documents available long before the court decision was made. In addition, our model predicts cases for the following month (i.e. the future), which is a hard task [7]. Therefore, it is nice to see the relatively high performance of our system with an accuracy of $75 \%$. By automatically highlighting critical sentences, and automatically updating every month, our system aims to offer a user-friendly web platform for legal professionals.

\section{References}

[1] Aletras N, Tsarapatsanis D, Preoţiuc-Pietro D, Lampos V. Predicting judicial decisions of the European Court of Human Rights: A natural language processing perspective. PeerJ Computer Science. 2016;2:e93.

[2] Sulea OM, Zampieri M, Vela M, Van Genabith J. Predicting the law area and decisions of French Supreme Court cases. arXiv preprint arXiv:170801681. 2017;

[3] Katz DM, Bommarito MJ, Blackman J. A general approach for predicting the behavior of the Supreme Court of the United States. PloS one. 2017;12(4):e0174698.

[4] Chen DL, Eagel J. Can machine learning help predict the outcome of asylum adjudications? In: Proceedings of the 16th edition of the International Conference on Articial Intelligence and Law; 2017. p. 237-240.

[5] Lage-Freitas A, Allende-Cid H, Santana O, de Oliveira-Lage L. Predicting Brazilian court decisions. arXiv preprint arXiv:190510348. 2019;

[6] O'Sullivan C, Beel J. Predicting the outcome of judicial decisions made by the European Court of Human Rights. arXiv preprint arXiv:191210819. 2019;.

[7] Medvedeva M, Vols M, Wieling M. Using machine learning to predict decisions of the European Court of Human Rights. Artificial Intelligence and Law. 2020;28(2):237-266.

[8] Greer S, Gerards J, Slowe R. Human rights in the Council of Europe and the European Union: Achievements, trends and challenges. Cambridge Studies in European Law and Policy. Cambridge University Press; 2018.

[9] Harris DJ, O’Boyle M, Bates E, Buckley C. Harris, O’Boyle \& Warbrick: Law of the European Convention on Human Rights. Oxford University Press, USA; 2014.

[10] Gerards J. General principles of the European Convention on Human Rights. Cambridge University Press; 2019. 